\title{
Cascaded logic gates in nanophotonic plasmon networks
}

Hong Wei', Zhuoxian Wang ${ }^{1}$, Xiaorui Tian'1, Mikael Käll2 \& Hongxing Xu'1,3

Optical computing has been pursued for decades as a potential strategy for advancing beyond the fundamental performance limitations of semiconductor-based electronic devices, but feasible on-chip integrated logic units and cascade devices have not been reported. Here we demonstrate that a plasmonic binary NOR gate, a 'universal logic gate', can be realized through cascaded OR and NOT gates in four-terminal plasmonic nanowire networks. This finding provides a path for the development of novel nanophotonic on-chip processor architectures for future optical computing technologies.

\footnotetext{
Beijing National Laboratory for Condensed Matter Physics and Institute of Physics, Chinese Academy of Sciences, Box 603-146, Beijing 100190, China. ${ }^{2}$ Department of Applied Physics, Chalmers University of Technology, Göteborg 412 96, Sweden. ${ }^{3}$ Division of Solid State Physics/The Nanometer Structure Consortium, Lund University, Box 118, Lund S-22100, Sweden. Correspondence and requests for materials should be addressed to H.X.X. (email: hxxu@iphy.ac.cn).
} 
$\mathrm{M}$ odern semiconductor-based electronics is rapidly approaching fundamental limits caused by interconnect delays and large heat generation. Photonic devices and circuits could potentially solve these problems, because photons have intrinsically higher information-carrying capacity and produce low heat loads ${ }^{1,2}$; but, the goal of all-optical computing has remained elusive. Previous developments have centred on two routes towards optical logic gates: the first one based on linear optical effects, such as interferometry $\mathrm{y}^{3-5}$, and the second one based on nonlinear processes, for example, tunable refractive indices or frequency mixing ${ }^{6-8}$. However, the diffraction limit of light has presented a fundamental obstacle for reducing the dimensions of optical logic components to the length scales of electronic devices in integrated circuits. During the past decade, it has been proposed that photonics based on surface plasmons, that is, quanta of collective electron oscillations strongly coupled to photons in metal nanostructures, constitute one of the most interesting avenues towards further scaling down of photonic devices ${ }^{9-18}$ and simultaneous integration with solid-state electronics ${ }^{19-21}$. Indeed, plasmonics is now actively pursued as a core methodology for nanoscale optical interconnects ${ }^{22-24}$.

We recently demonstrated a complete family of basic logic functions, that is, AND, OR and NOT gates, in simple photonic networks composed of interconnected silver nanowires ${ }^{25}$. The logic operations were realized by utilizing polarization and phase-dependent interference between plasmon beams propagating through the wire network. But more complex logic functions and functional photonic circuits rely on the possibility of cascading the basic gates. A particularly important step in this direction is the construction of the so-called universal gates, that is NAND and NOR gates, which can be combined to implement essentially any logic operation.

Here we show that it is possible to cascade OR and NOT gates to realize a plasmonic inverse NOR gate in a four-terminal branched silver nanowire network. We use quantum dot (QD) near-field imaging to trace the plasmon wave packets through the branched network and to demonstrate precise control of optical interferences at the nanoscale. We believe that these observations and results may pave the way for the development of tomorrow's plasmon circuits for on-chip integrated optical computing.

\section{Results}

Cascaded plasmonic OR and NOT gates for NOR gate. Figure 1a illustrates the schematics of cascaded plasmonic OR and NOT gates for the universal logic gate NOR. It has recently been demonstrated that the interferences of two plasmon beams in single-branched $\mathrm{Ag}$ NW structure can function as OR or NOT gates ${ }^{25}$. Here we arranged Ag NWs (Supplementary Fig. S1) with a micromanipulator to construct a four-terminal NW structure composed of a primary NW and two-branch NWs as shown in Figure 1b. The typical process for the construction of a NW network is shown in Supplementary Figure S2 for example. The first branch NW with the terminal marked as 'I2' and the first half-part of the primary NW with terminal marked as 'I1' form the first branched structure, which is designed to function as an OR gate. The second branch NW with the terminal marked as ' $C$ ' and the last-half part of the primary NW forms the second branched structure, which is designed to be a NOT gate.

The propagating plasmons in the network can be launched through the excitation at the terminals marked as I1, I2 and C, or any combinations of them. It was known that the propagating plasmons in Ag NWs can be launched by laser excitation at the NW terminals or other discontinuities, such as nanoparticle-nanowire junctions ${ }^{26-28}$. In single-branched NW structures composed of a primary NW and a branch NW, the plasmons on the NW can also be excited by laser illumination at the branch tip $^{25,29}$. If two plasmon beams are generated on the primary NW a

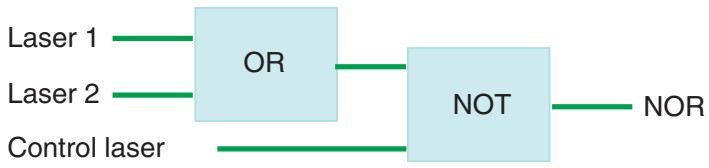

b

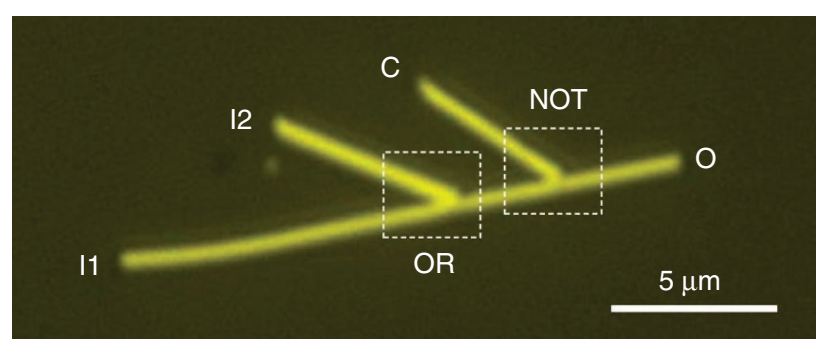

C

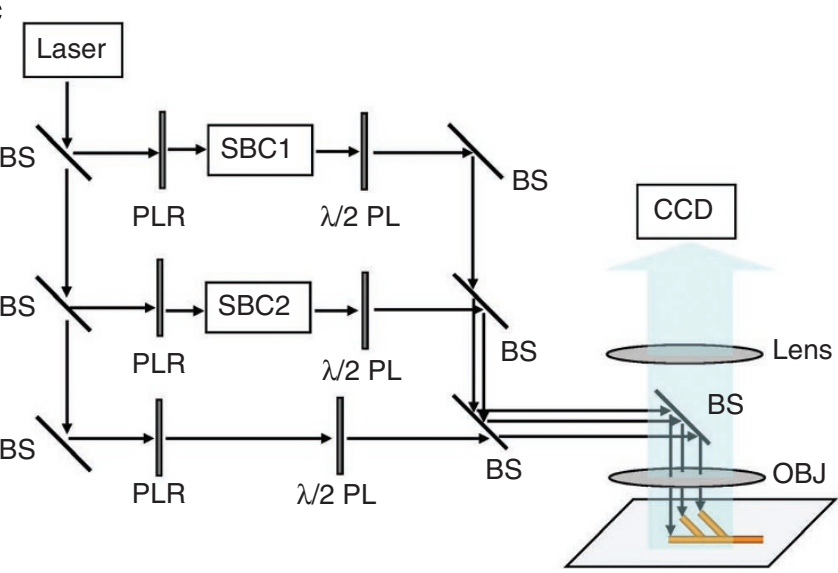

Figure 1 | Cascaded logic gates for NOR gate and the experimental setup. (a) Schematic illustration of logic gate NOR built by cascaded OR and NOT gates. (b) Optical image of the designed Ag NW structure. (c) Schematics of experimental setup. $\mathrm{BS}$, beamsplitter; $\mathrm{OBJ}$, objective; $\mathrm{PLR}$, polarizer; $\mathrm{SBC}$, Soleil-Babinet compensator; $\lambda / 2 \mathrm{PL}$, half-wave plate.

by excitation at the primary NW tip and the branch tip, these two beams will interfere on the NW and modulate the near field distribution and the output scattering intensity. Plasmonic OR or NOT gates can be obtained by tuning the intensity, phase and polarization of the incident lasers. With same mechanism, in cascade structures as in Figure 1b, the first branched Ag NW structure can function as OR gate by tuning the incident lasers at its input terminals, whereas the second branched structure functions as NOT gate to invert the output 'state' of the first structure by tuning the incident laser at its branch tip C.

The optical setup is schematically shown in Figure 1c. A 633-nm laser is divided into three beams by beam splitters, and the polarization of each beam can be controlled by half-wave plates. SoleilBabinet compensators are inserted into two of the beams, which can control the phase difference of these beams. These three beams were focused on the three input terminals of plasmonic NOR gate as shown in Figure 1b.

If any two excitation beams are incident on two different terminals, the plasmons launched by these two laser beams will interfere in the branched NW structures. The output intensity will depend on the phase difference of these two laser beams ${ }^{25}$. By continuously changing the phase of one incident laser with a compensator, the intensity of the emitted light at the output end marked as ' $\mathrm{O}$ ' will oscillate between the maximum value and the minimum value. Figure $2 \mathrm{a}$ shows the case for two inputs from the I1 and $\mathrm{C}$ terminals. The phase difference is controlled by tuning the compensator 1 in 
a

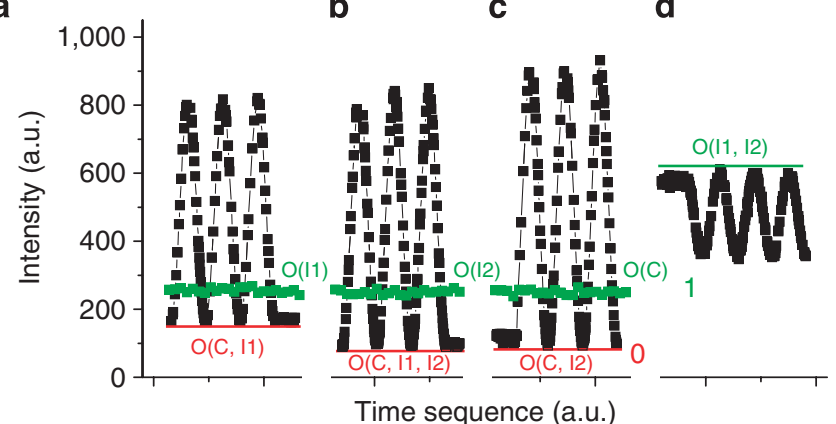

e

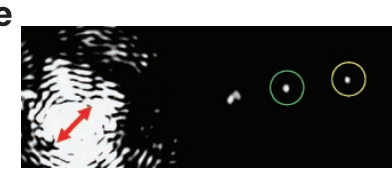

f

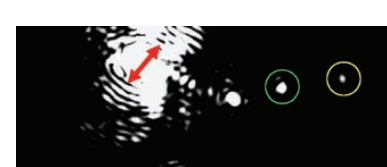

g

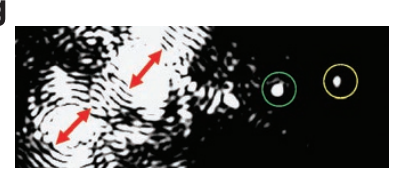

h

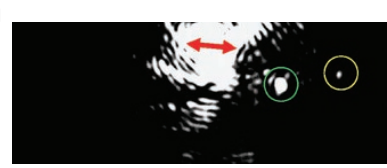

i

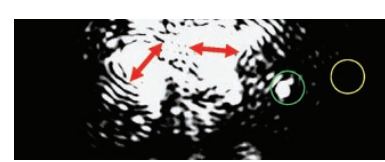

j

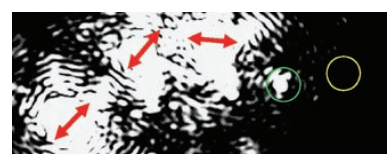

Figure 2 | Output intensity at the $\mathbf{O}$ end for different inputs versus the time sequence as the incident phases are changed. (a-d) The output intensity for the inputs from terminals $(C, 11),(C, 11,12),(C, 12)$ and $(11$, 12). The red lines emphasize the output intensity for the NOR operation. The green line emphasizes the corresponding output intensity for $(11,12)$

(e-g) The scattering images showing the OR operations. (h) The scattering image when only $\mathbf{C}$ was enabled. $(\mathbf{i}, \mathbf{j})$ The scattering images for the NOR operations, corresponding to a NOT function acting on $(\mathbf{f}, \mathbf{g})$. The red arrows show the incident laser polarization. The green and yellow circles mark the $\mathrm{C}$-branch junction and the $\mathrm{O}$ terminal, respectively.

the light path for the I1 input. When the output intensity gets to the minimal value, further tuning of the compensator was stopped and the phase from I1 was fixed. A third laser beam is then applied to terminal I2. All three plasmon beams will interfere on the primary wire. By tuning the phase of the I2 input with another compensator, the output intensity will oscillate between a new minimum and maximum value as shown in Figure $2 b$. When the output intensity reached minimum, the $\mathrm{I} 2$ phase was fixed by stopping tuning of the corresponding compensator 2. At this time, the I1 beam was blocked so that only I 2 and C interfere. It is found that the output is also the minimum to compare with other different interferences between $\mathrm{I} 2$ and $\mathrm{C}$ by changing the phase of I 2 as shown in Figure $2 \mathrm{c}$. That is to say, there is a particular given phase of I1 and $\mathrm{I} 2$ for which the outputs $\mathrm{O}(\mathrm{C}, \mathrm{I} 1), \mathrm{O}(\mathrm{C}, \mathrm{I} 2)$, and $\mathrm{O}(\mathrm{C}, \mathrm{I} 1, \mathrm{I} 2)$ are simultaneously minimized while the output $\mathrm{O}(\mathrm{I} 1, \mathrm{I} 2)$ is close to maximum as shown in Figure $2 \mathrm{~d}$.

If we define a scattering intensity from the output terminal of $>200$ as the ' 1 ' or 'ON' state, for example, the value above the green dot lines $(\mathrm{O}(\mathrm{I} 1), \mathrm{O}(\mathrm{I} 2), \mathrm{O}(\mathrm{C})$ ) in Figure $2 \mathrm{a}-\mathrm{c}$, and the intensity $<200$, for example, the red lines as ' 0 ' or 'OFF' state, then all of the two inputs interferences and the three inputs interferences result in ' 0 ' output for certain input phases. To illustrate the optical binary logic NOR gate, we treat terminal I1 and I2 as the input ends for the signals, and terminal $\mathrm{C}$ as the control end. For any input from either

Table 1 | Outputs of logic gates.

\begin{tabular}{|c|c|c|c|c|c|}
\hline \multicolumn{3}{|c|}{ Input } & \multicolumn{3}{|c|}{ Output } \\
\hline 11 & 12 & C & O1=I1 OR I2 & $(01, C)=$ NOT 01 & $0=I 1$ NOR I2 \\
\hline 0 & 0 & 1 & 0 & 1 & 1 \\
\hline 0 & 1 & 1 & 1 & 0 & 0 \\
\hline 1 & 0 & 1 & 1 & 0 & 0 \\
\hline 1 & 1 & 1 & 1 & 0 & 0 \\
\hline
\end{tabular}

I1 or I2 only, the control $\mathrm{C}$ will invert it, that is, invert ' 0 ' to ' 1 ', or ' 1 ' to ' 0 '. Hence, if we have input variables from (I1, I2): $(0,0),(1,0)$, $(0,1),(1,1)$, the output is $1,0,0,0$, respectively. That is the Boolean logic gate NOR, as shown in Table 1 .

To demonstrate that this plasmonic NOR gate is actually caused by cascading a plasmonic OR gate and a NOT gate, the scattering images for different inputs are shown in Figure $2 \mathrm{e}-\mathrm{j}$. Figure $2 \mathrm{e}-\mathrm{g}$ clearly shows the outputs are ON when either or both I1 and I2 are $\mathrm{ON}$, thus functioning as an $\mathrm{OR}$ gate. When control $\mathrm{C}$ is enabled, and I1 and I2 are disabled, as shown in Figure $2 \mathrm{~h}$, the output is ON, which means a NOT function. When this NOT function acts on the OR gate, then it will invert the output to be OFF, when either or both I1 and $\mathrm{I} 2$ are ON, as shown in Figure 2i,j. From Figure 2e-j, we firstly demonstrate OR gate, then let NOT gate function with $\mathrm{C}$ input enabled, and the overall effect is the NOR gate. Therefore, we have illustrated that the plasmonic NOR gate in this four-terminal structure designed here is composed of cascaded plasmonic OR and NOT gates. The outputs of different gates for each combination of the three input variables are shown in Table 1.

When the principle of cascaded plasmonic devices for NOR gate is understood, we can design different NWs network structures to realize NOR function based on the same principle. Supplementary Figure S3 shows another example of plasmonic NOR gate in a different four-terminal NW structure, that is, two-branch NWs locating at the different sides of the primary NW, but with the same logic function as shown in Table 1 as well.

QD imaging reveals near-field distribution on NWs. To understand the mechanism of cascaded plasmonic logic devices, we use QD fluorescence to image the electromagnetic (EM) near-field distribution of the propagating plasmons on metal $\mathrm{NWs}^{25}$. Figure $3 \mathrm{a}$ shows the QD fluorescence image by a wide field excitation, which indicates an even distribution of QD coating on the NWs. The white dashed lines show the outline of the branch NWs. When the 633nm laser was focused on only the I1 terminal with polarization of about $40^{\circ}$, as indicated by the white arrow in Figure 3b, corresponding to the scattering image shown in Figure 2e, the QD fluorescence image shows that the propagating plasmon energy has 'node-like' zigzag near-field distribution pattern. This pattern is caused by the superposition of different propagating plasmon modes induced by the incident laser at certain polarization, which modulates electric field distribution along the NW25,30,31.

The white-dotted curved lines mark the outline of the EM field nodes at the two connection junctions between the branches and the main wire. It is interesting that the node position of zigzag pattern with strong EM near-field intensity overlapped with the position of the junction between the main wire and the C-branch wire. When the laser with the same polarization was focused on the terminal I2, the 'node-like' zigzag near-field pattern with strong EM near-field intensity again overlapped with the position of the connect junction of the $\mathrm{C}$ branch as shown in Figure $3 \mathrm{c}$, which corresponds to the scattering image of Figure $2 \mathrm{f}$. When these two lasers were focused on the I1 and I2 terminals simultaneously with the same phases as in Figure 2g, the zigzag pattern with strong EM near-field intensity also overlaps with the C-branch junction as shown in Figure 3d. 


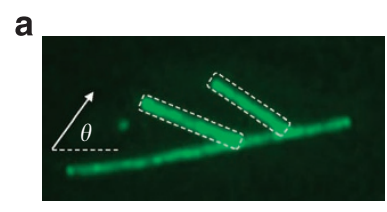

b

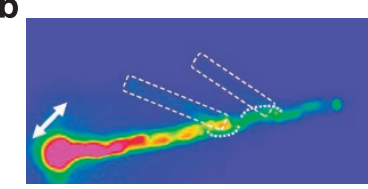

C

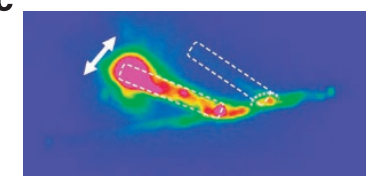

d

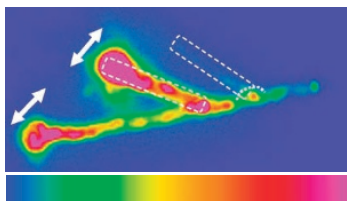

Min

$\operatorname{Max}$ e

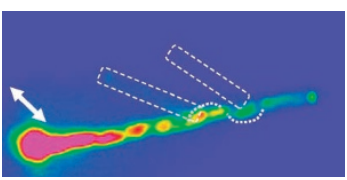

f

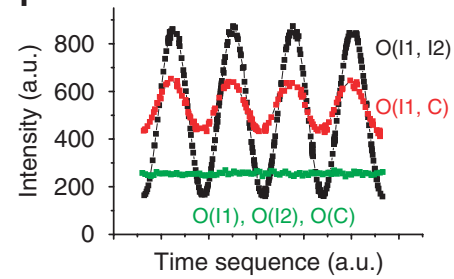

g

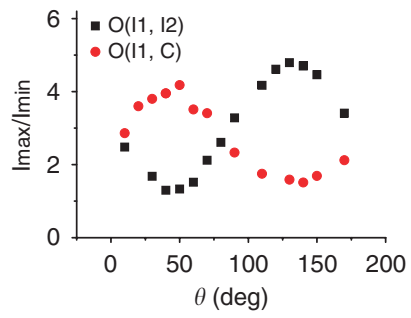

Figure 3 | Dependence of plasmon interference on incident polarizations. (a) QD fluorescence image by wide-field illumination. Dashed white lines show the outline of the branch NWs. (b-e) QD fluorescence images with excitation at different terminals with polarization indicated by the white arrows. (f) The output intensity at terminal $\mathrm{O}$ as a function of the phase difference between two laser beams. Green: the output intensity when only one input $(11,12$, or $\mathrm{C})$ is applied. Black: the output intensity resulting from two incident beams (I1 and 12). Red: the output intensity resulting from two incident beams ( 11 and $\mathrm{C}$ ). The incident polarizations at 11 and 12 terminals used for this set of measurement corresponds to the polarizations shown in $(\mathbf{e}, \mathbf{c})$. The incident polarization at $\mathbf{C}$ terminal is the same as in Figure 2. (g) Interference depth of $(11,12)$ and $(11, C)$ as a function of polarization angle $\theta$ of the input laser at the 11 terminal. The definition of $\theta$ is shown in (a). The polarizations of $\mathrm{I} 2$ and $\mathrm{C}$ are kept the same as those in Figure 2.

In Figure $2 \mathrm{e}-\mathrm{g}$, we have demonstrated the function of plasmonic OR gate. As will be seen, the cascaded NOT gate will work when the zigzag pattern with the locally strongest EM field distribution overlaps with the C-branch junction. To make NOT gate work well, this kind of near-field distribution at the branch junction position is crucial to obtain sufficient interference between two plasmon beams (one in the main wire, and the other one in the branch) to make the output intensity sufficiently small as shown in Figure 2i,j, or the minimum values of $(\mathrm{C}, \mathrm{I} 1),(\mathrm{C}, \mathrm{I} 2)$ and $(\mathrm{C}, \mathrm{I} 1, \mathrm{I} 2)$ in Figure $2 \mathrm{a}-\mathrm{c}$.

Unlike the plasmonic NOT gate, significant interference is not required for the plasmonic OR gate. As shown in Figure 2d, the output scattering intensity $\mathrm{O}(\mathrm{I} 1, \mathrm{I} 2)$ can be somehow modulated by the interference between two plasmon beams I1 and I 2 at different phases, but not as strongly as the interference between either or both of these two plasmon beams, and the plasmon beam from the $\mathrm{C}$ terminal is shown in $\mathrm{O}(\mathrm{C}, \mathrm{I} 1), \mathrm{O}(\mathrm{C}, \mathrm{I} 2)$ and $\mathrm{O}(\mathrm{C}, \mathrm{I} 1, \mathrm{I} 2)$ of Figure $2 \mathrm{a}-\mathrm{c}$, where the inverse logic function is needed to perform the NOR operation. If we check the zigzag pattern of EM near-field distribution shown in Figure 3b, the locally most intense part is located at the opposite side of the junction between the primary wire and the branch wire (I2). Evidently, strong interference requires that two beams should meet closely at the beginning, that is, the wave packet position with strong EM intensity should overlap with the connecting junction. Hence, the degree of such overlapping will determine how well the beams interfere. It should be noted that,

although the junction of the $\mathrm{C}$ branch in Figure $3 \mathrm{~b}-\mathrm{d}$ is located at the stronger EM field position, it is deviated from the centre of the EM field packet in Figure $3 \mathrm{~b}$ compared with Figure 3c,d. This may explain why the destructive interference for $\mathrm{O}(\mathrm{C}, \mathrm{I} 1)$ is not as good as for $\mathrm{O}(\mathrm{C}, \mathrm{I} 2)$ and $\mathrm{O}(\mathrm{C}, \mathrm{I} 1, \mathrm{I} 2)$, that is, the lowest value of $\mathrm{O}(\mathrm{C}, \mathrm{I} 1)$ is higher than lowest values of $\mathrm{O}(\mathrm{C}, \mathrm{I} 2)$ and $\mathrm{O}(\mathrm{C}, \mathrm{I} 1, \mathrm{I} 2)$.

To further verify our point about the relation between how strong the interference is and EM field intensity distribution, the EM near-field distribution pattern in the main wire is varied by changing the I1 input polarization. For example, rotating the laser polarization for about $130^{\circ}$, as shown in Figure 3 e, suddenly changes the zigzag EM near-field distribution pattern from that in the Figure 3b. In Figure 3e, the intense EM field packet of the zigzag pattern is completely moved away from the C-branch junction, while the $\mathrm{I} 2$ branch junction overlaps with an intense EM field packet. Comparing with Figure 2a, the interference between $\mathrm{C}$ and I1 becomes much less strong, as shown in Figure $3 \mathrm{f}$ (red curve), while the interference between I1 and I2 becomes much stronger (black curve) compared with Figure 2d.

We define how well the plasmon beams interfere, or the interference depth, as the ratio of maximum and minimum scattering intensity (Imax/Imin), when the phase of two plasmon beams differs by $\pi$. Figure $3 g$ shows this ratio versus the incident polarization angle at the terminal I1. For the strongest interference between $\mathrm{C}$ and I1, occurring when the EM wave packet overlaps with the C-branch junction for the incident polarization as in Figure 3b, the interference between I1 and I 2 is the weakest in our device demonstrated here, and the vice versa. Hence, the interference depth can be tuned by shifting the zigzag EM wave packet on or off the branch junctions with different incident polarization on the primary wire. This clearly shows that the zigzag EM field pattern on the wire can indeed function as 'switch' to control the interference of two plasmon beams in the NW network.

It should be noted that the reverse incident polarization dependence of interference depth for (I1, I2) and (I1, C) observed here just occasionally happens for this structure, but it is not a required condition to realize the NOR function. As the NOR function is relied on the NOT function to reverse the state at the $\mathrm{C}$ junction, there should be no relevancy to the interference depth of (I1, I2). In Supplementary Figure S4, the different incident polarization dependence of interference depth for (I1, I2) and (I1, C) is observed, which shows almost the same polarization dependence. Hence, to realize NOR operation, the incident polarization in the primary NW at I1 is only required to guarantee the stronger interference at the $\mathrm{C}$ junction, that is, the plasmon wave packet overlapping largely with the $\mathrm{C}$ junction. This incident polarization is usually in a range of tens of degrees.

\section{Discussion}

We have demonstrated that cascaded plasmonic OR and NOT gates can be used to form a plasmonic NOR gate in a four-terminal nanowire network by multiple-beam plasmon interference. As cascading plasmonic devices are now possible, more complex functions can be realized by the precise design of complex plasmonic waveguide networks. NOR and NAND gates, the so-called universal logic gates, are the important elements of Boolean logics and are crucial to modern electronic circuits, because any Boolean logic gate can be constructed from a suitable network of NOR gates only, or NAND gates only. Similarly, plasmonic NOR gates demonstrated here could have key roles to constitute a logical formal system of Boolean logic in future optical computing. Moreover, plasmonic devices of the type discussed here can be further miniaturized and it is likely that improvement of the materials and wire interconnects will greatly reduce signal losses, thereby maintaining signal thresholds for the logic operations. This could allow for on-chip integration of a large number of cascaded subwavelength logic units 
for complex optical processing. Finally, our plasmonic structures allow for coherent control of optical quantum interferences and may therefore have a role in the development of future photonic quantum computation devices ${ }^{32-34}$.

\section{Methods}

Synthesis of Ag NWs and the construction of NW structures. The Ag NWs were synthesized by a wet chemical method as reported in the literature ${ }^{35}$. The products of the chemical reaction were washed with ethanol by centrifugation and redispersed in ethanol. The diameter of the Ag NWs is between 200 and $350 \mathrm{~nm}$ and the length is a few to tens of microns. Nanowires of such diameters have good elasticity and good toughness, which facilitate the manipulation of these NWs. A micromanipulator (MMO-202ND, Narishige) is used to manipulate the NWs and build the designed structures without having the NWs damaged.

After the branched NW structure was built, an $\mathrm{Al}_{2} \mathrm{O}_{3}$ layer of $30 \mathrm{~nm}$ was deposited by atomic layer deposition. Then, QDs with emission wavelength centred at $655 \mathrm{~nm}$ were spincoated onto the sample.

Optical measurements. The optical measurements were based on an Olympus BX51 microscope equipped with a piezo stage of high positioning accuracy and a CCD camera. The $633 \mathrm{~nm}$ laser was focused on the sample by a $\times 100$ (NA 0.95 ) objective. The light from the sample was collected by the same objective and directed to the CCD camera to get the images. For the QD fluorescence imaging, a $633 \mathrm{~nm}$ long pass edge filter was used to block the laser.

\section{References}

1. Kirchain, R. \& Kimerling, L. A roadmap for nanophotonics. Nat. Photonics 1, 303-305 (2007).

2. Engheta, N. Circuits with light at nanoscales: optical nanocircuits inspired by metamaterials. Science 317, 1698-1702 (2007).

3. Kwong, S. K., Rakuljic, G. A. \& Yariv, A. Real-time image subtraction and exclusive or operation using a self-pumped phase conjugate mirror. Appl. Phys. Lett. 48, 201-203 (1986).

4. Ogusu, M., Tanaka, S. \& Kuroda, K. Optical logic operations using 3-beam phase-conjugate interferometry. Jpn. J. Appl. Phys. 29, L1265-L1267 (1990).

5. Zhang, Y. L., Zhang, Y. \& Li, B. J. Optical switches and logic gates based on self-collimated beams in two-dimensional photonic crystals. Opt. Express 15, 9287-9292 (2007)

6. Fainman, Y., Guest, C. C. \& Lee, S. H. Optical digital logic operations by 2-beam coupling in photorefractive material. Appl. Opt. 25, 1598-1603 (1986).

7. Almeida, V. R., Barrios, C. A., Panepucci, R. R. \& Lipson, M. All-optical control of light on a silicon chip. Nature 431, 1081-1084 (2004).

8. Xu, Q. F. \& Lipson, M. All-optical logic based on silicon micro-ring resonators. Opt. Express 15, 924-929 (2007)

9. Maier, S. A. et al. Plasmonics-a route to nanoscale optical devices. Adv. Mater 13, 1501-1505 (2001).

10. Tuchscherer, P. et al. Analytic coherent control of plasmon propagation in nanostructures. Opt. Express 17, 14235-14259 (2009).

11. Schnell, M. et al. Controlling the near-field oscillations of loaded plasmonic nanoantennas. Nat. Photonics 3, 287-291 (2009).

12. Curto, A. G. et al. Unidirectional emission of a quantum dot coupled to a nanoantenna. Science 329, 930-933 (2010).

13. Barnes, W. L., Dereux, A. \& Ebbesen, T. W. Surface plasmon subwavelength optics. Nature 424, 824-830 (2003).

14. Lal, S., Link, S. \& Halas, N. J. Nano-optics from sensing to waveguiding. Nat. Photonics 1, 641-648 (2007).

15. Polman, A. Plasmonics applied. Science 322, 868-869 (2008).

16. Gramotnev, D. K. \& Bozhevolnyi, S. I. Plasmonics beyond the diffraction limit. Nat. Photonics 4, 83-91 (2010).

17. Schuller, J. A. et al. Plasmonics for extreme light concentration and manipulation. Nat. Mater. 9, 193-204 (2010).

18. Chen, X. W., Sandoghdar, V. \& Agio, M. Highly efficient interfacing of guided plasmons and photons in nanowires. Nano Lett. 9, 3756-3761 (2009).

19. Ozbay, E. Plasmonics: merging photonics and electronics at nanoscale dimensions. Science 311, 189-193 (2006).
20. Zia, R., Schuller, J. A., Chandran, A. \& Brongersma, M. L. Plasmonics: the next chip-scale technology. Mater. Today 9, 20-27 (2006)

21. Brongersma, M. L. \& Shalaev, V. M. The case for plasmonics. Science 328, 440-441 (2010).

22. Kim, J. T. et al. Chip-to-chip optical interconnect using gold long-range surface plasmon polariton waveguides. Opt. Express 16, 13133-13138 (2008).

23. Kim, J. T. CMOS-compatible hybrid plasmonic waveguide for subwavelength light confinement and on-chip integration. IEEE Photon. Technol. Lett. 23, 206-208 (2011).

24. Alu, A. \& Engheta, N. Wireless at the nanoscale: optical interconnects using matched nanoantennas. Phys. Rev. Lett. 104, 213902 (2010).

25. Wei, H. et al. Quantum dot-based local field imaging reveals plasmon-based interferometric logic in silver nanowire networks. Nano Lett. 11, 471-475 (2011).

26. Ditlbacher, H. et al. Silver nanowires as surface plasmon resonators. Phys. Rev. Lett. 95, 257403 (2005).

27. Sanders, A. W. et al. Observation of plasmon propagation, redirection, and fan-out in silver nanowires. Nano Lett. 6, 1822-1826 (2006).

28. Knight, M. W. et al. Nanoparticle-mediated coupling of light into a nanowire. Nano Lett. 7, 2346-2350 (2007)

29. Li, Z. P., Zhang, S. P., Halas, N. J., Nordlander, P. \& Xu, H. X. Coherent modulation of propagating plasmons in silver-nanowire-based structures. Small 7, 593-596 (2011).

30. Fang, Y. R. et al. Branched silver nanowires as controllable plasmon routers. Nano Lett. 10, 1950-1954 (2010).

31. Li, Z. P. et al. Correlation between incident and emission polarization in nanowire surface plasmon waveguides. Nano Lett. 10, 1831-1835 (2010).

32. Politi, A., Cryan, M. J., Rarity, J. G., Yu, S. Y. \& O’Brien, J. L. Silica-on-silicon waveguide quantum circuits. Science 320, 646-649 (2008).

33. Matthews, J. C. F., Politi, A., Stefanov, A. \& O’Brien, J. L. Manipulation of multiphoton entanglement in waveguide quantum circuits. Nat. Photonics 3 , 346-350 (2009)

34. Peruzzo, A., Laing, A., Politi, A., Rudolph, T. \& O’Brien, J. L. Multimode quantum interference of photons in multiport integrated devices. Nat. Comm. 2, $224(2011)$

35. Sun, Y. G. \& Xia, Y. N. Large-scale synthesis of uniform silver nanowires through a soft, self-seeding, polyol process. Adv. Mater. 14, 833-837 (2002)

\section{Acknowledgments}

We thank Nathaniel Grady for the help in the preparation of the manuscript, Peter Nordlander and Naomi Halas for valuable discussions, the Laboratory of Microfabrication in Institute of Physics of CAS for experimental support. This work was supported by NSFC Grants (10625418, 10874233 and 11004237), MOST Grants (2006DFB02020 and 2009CB930700), 'Bairen Project' and 'Knowledge Innovation Project' (KJCX2-EW-W04) of CAS

\section{Author contributions}

H.X.X. conceived the idea and supervised the project. H.W. carried out the experiment. H.W., Z.X.W. and X.R.T. prepared the samples. H.W. and H.X.X. analysed the data. H.W., H.X.X. and M.K. wrote the manuscript.

\section{Additional information}

Supplementary Information accompanies this paper at http://www.nature.com/ naturecommunications

Competing financial interests: The authors declare no competing financial interests.

Reprints and permission information is available online at http://npg.nature.com/ reprintsandpermissions/

How to cite this article: Wei, H. et al. Cascaded logic gates in nanophotonic plasmon networks. Nat. Commun. 2:387 doi: 10.1038/ncomms1388 (2011).

License: This work is licensed under a Creative Commons Attribution-NonCommercial Share Alike 3.0 Unported License. To view a copy of this license, visit http:// creativecommons.org/licenses/by-nc-sa/3.0/ 